\title{
Technical Developments and Principal Results of Vertical Feeding Method for GaSb and GaInSb Alloys
}

\author{
J. Vincent ${ }^{1 *}$, C. Díaz-Guerra ${ }^{2}$, J. Piqueras ${ }^{2}$, E. Diéguez ${ }^{1}$ \\ ${ }^{1}$ Departamento de Física de Materiales, Facultad de Ciencias, Universidad Autónoma de Madrid, \\ 28049 Madrid, Spain e-mail: julien.Vincent@uam.es \\ ${ }^{2}$ Departamento de Física de Materiales, Facultad de Ciencias Físicas, Universidad Complutense de \\ Madrid, E-28040, Madrid, Spain.
}

\begin{abstract}
In this work, the experimental set-up of the Vertical Feeding Method (VFM) which has been used for the preparation of GaSb and GaInSb materials is presented. The different elements of the set-up are detailed as well as the preparation process. The different configurations that have been used for material production are briefly described and the principal results are summarized. In the case of Te-doped GaSb materials, the study, focused on the grain size and structure of the as-grown materials, showed that single crystals can be obtained with the VFM. In the case of GaInSb materials, the study, focused on the spatial indium distribution in the solid phase prepared with the different VFM configurations, showed that an effective band edge reduction can be achieved.
\end{abstract}

Keywords: Bulk crystal growth, III-V compounds, Doping in III-V semiconductors. PACS: $81.05 \mathrm{Ea}, 81.10 \mathrm{Fq}, 81.30 \mathrm{Fb}, 61.72 \mathrm{Vv}$

\section{INTRODUCTION}

Low band gap materials like GaSb and GaInSb concentrated alloys are of considerable interest for the development of thermophotovoltaic (TPV) technology. Pure GaSb is intrinsically p-type and requires doping with elements such as Te, Se, etc to be used in TPV applications. Doping generally results in a reduction of the quality and monocrystallinity of the material and several authors have explored the possibility to use TPV cells manufactured on polycrystalline Te-doped GaSb wafers [1,2] to reduce production cost. In the case of GaInSb concentrated alloys, the possibility to use substrates with a tailored band gap and lattice parameter as a function of the alloy composition is still constrained by the low quality of the materials grown by classical bulk growth techniques.

In order to enhance the crystalline quality and to control the solute segregation phenomenon, a crystal growth technique based on the feeding principle has been proposed. This technique which is named as Vertical Feeding Method (VFM) requires the use of two vertical crucibles with an independent control of the temperature of each crucible and a controlled dispatching of the feed material from one crucible to the

CP890, Thermophotovoltaic Generation of Electricity, Seventh Conference

edited by C. Algora and V. Corregidor

(C) 2007 American Institute of Physics 978-0-7354-0392-5/07/\$23.00 
other. In order to give the possibility to implement the VFM, we present here the technical solutions retained to achieve the experimental set-up and the principal results obtained for GaSb and GaInSb materials as an illustration of the VFM potentialities.

In the first part of the work, the principle of feeding techniques is briefly reminded and the different elements of the VFM set-up are presented. In the second part, the different configurations that have been used for material production are briefly described and the principal results are summarized. In the case of Te-doped GaSb materials, the study demonstrates the possibility to control the segregation and the grain size and structure using an appropriate VFM configuration. In the case of GaInSb materials, the study demonstrates that solid solutions with different indium spatial distributions have been obtained with the different VFM configurations.

\section{PRINCIPLE OF FEEDING TECHNIQUES}

Different technical solutions have been studied to improve the quality and homogeneity of the crystals grown from the melt by classical techniques. The use of an alternating magnetic field located in the vicinity of the solid-liquid interface [3], of an internal baffle moving at a constant distance from the interface [4] or the double crucible Czochralski technique [5] have been proposed with the aim of preserving the characteristic of the liquid (concentration, temperature) in a thin layer close to the Solid/Liquid interface, allowing to improve the materials homogeneity. With these methods, most of the melt is used as a feed for the thin liquid layer and a special design is required to limit the interactions between the thin layer and the remaining melt [6]. One possibility to eliminate these interactions is to physically separate the growing melt and the feed material as it is usual in casting techniques [7-9] by using a two crucible set-up with a controlled dispatching of the feed material from one crucible to the other.

\section{EXPERIMENTAL SET-UP}

As shown on figure 1, the VFM set-up developed in our laboratory is composed by three main parts: a two-zone furnace, a crucibles set-up and a pressure control system. The two-zone furnace has been designed to control independently the melting of the feed material and the temperature of the growing material. The upper zone is designated as the feeding chamber while the lower one as the growth chamber.

The furnace consists in two Thermocoax ${ }^{\circledR}$ heating elements which enable local heating, each of them equipped with a Eurotherm ${ }^{\circledR}$ temperature controller with temperatures measured by type $\mathrm{K}$ thermocouples. The thermal gradient imposed by the furnace in the growth chamber ranges between 10 and $20 \mathrm{~K} / \mathrm{cm}$. 


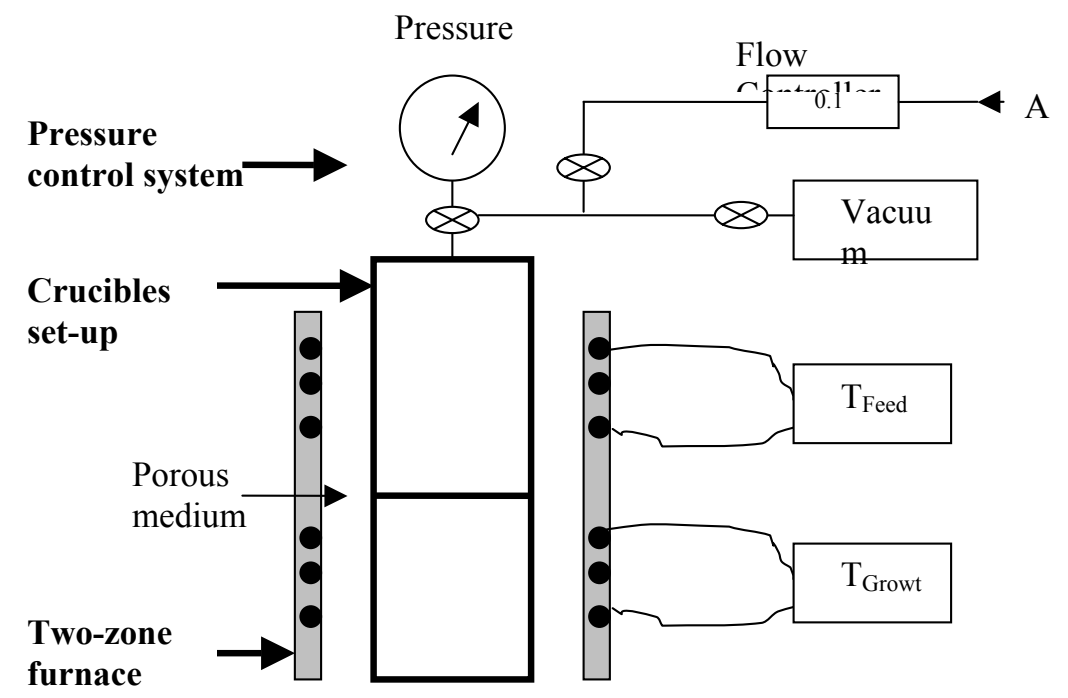

FIGURE 1. Schematic representation of the VFM set-up showing the two-zone furnace, the crucible set-up and the pressure control system.

For simplicity reasons, the crucibles set-up is entirely made with quartz material and as shown on figure 2, it is composed by three elements: i) a feeding crucible that consists in a quartz tube ended by a porous quartz plate, ii) a growth crucible where the solidification takes place and iii) an "outer" ampoule where the growth crucible is placed. Depending on the temperature and weight of the melted material and on the porosity of the medium, the feed materials can or not flow through the quartz plate. For practical uses, the feed material should not flow freely but in a controlled way; this point has been achieved thanks to the addition of a pressure control system that is described in the following section. Medium porosity plates showed an effective control of the feeding rate, and the porosities used in all experiments were 150-90 or 90-40 microns. As shown on figure 2, the crucible set-up is assembled using commercial vacuum spanners.

As schematized on figure 1, the pressure control system is composed by a flow controller connected to an Argon bottle, a vacuum pump, an overpressure measurement and vacuum keys to open and close the different paths. 


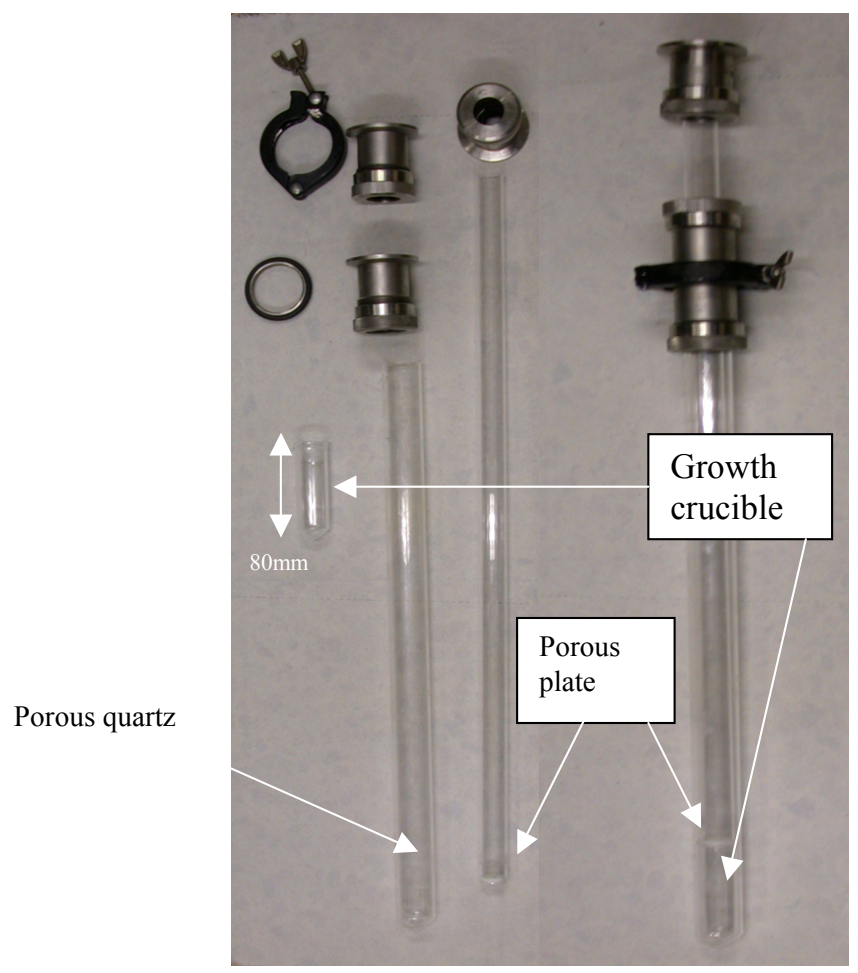

FIGURE 2. Photographs showing the crucible set-up composed by an outer ampoule, a feeding and a growth crucibles, before and after assembling with the vacuum spanners.

The flow-meter is a compact commercial Mass Stream series D-51 flow meter from $\mathrm{M}+\mathrm{W}$ Instruments $\mathrm{GmgH}$ that operates on the principle of heat transfer by sensing the delta-T along a heated section of a capillary tube. The pressure control system that permits to increase continuously the pressure in the feeding crucible is directly connected to the top end of the feeding crucible using commercial vacuum spanners. Experiments showed that the feeding rate, which is defined as the ratio between the total volume of the feed material and the feeding time, depends on the rate of pressure increase and once a critical pressure is exceeded, the feed material drops from one crucible to the other in a steady state way. The critical pressure is highly dependent on the material viscosity (which is a function of temperature [10]) and for the experiments described in this work, it ranged between 200 and 800 Torr.

\section{EXPERIMENTAL PROCEDURE}

Once the ampoules set-up is cleaned, prepared and loaded with material presynthesized by the Vertical Bridgman technique, it is introduced in the two-zone furnace and connected to the pressure control system to perform a vacuum of about $10^{-2}$ Torr. The feeding chamber temperature $T_{\text {feed }}$ is then increased during $6 \mathrm{~h}$ up to 
few degrees above the melting temperature $T_{\mathrm{m}}$ while the growth chamber temperature is increased to the desired growth temperature $T_{\text {growth. }}$. When the load material is melted, feeding is achieved at constant rate by increasing continuously the pressure in the feeding crucible until the liquid begins to flow from one crucible to the other. Once feeding is completed and the whole ingot crystallized, the furnace temperature is kept constant for $2 \mathrm{~h}$ and then slowly cooled down to room temperature at $5 \mathrm{~K} / \mathrm{h}$.

TABLE 1. Acronyms of the different configurations corresponding to the experimental conditions of the VFM experiments.

\begin{tabular}{|c|c|c|c|}
\hline \multicolumn{1}{|l|}{$\mathbf{V}_{\text {feed }}(\mathbf{m L} / \mathbf{m i n})$} & $\mathbf{2 0 - 1 0}$ & $\mathbf{5 - 1}$ & $\mathbf{1 - 0 . 1}$ \\
\hline $\mathbf{7 0 - 1 2 0}$ & & & \\
\hline $\mathbf{3 0 0 - 3 2 0}$ & Low Casting \\
& $(\mathrm{LC}-)$ & High Feeding (HFeed- & $\begin{array}{c}\text { Low Feeding } \\
\text { (LFeed-) }\end{array}$ \\
\hline $\mathbf{5 0 0 - 5 2 0}$ & High Casting & High Splat cooling & \\
& (HC-) & Low Splat cooling & \\
& & (HSp-) & \\
\hline
\end{tabular}

The two operative parameters of the VFM are the feeding velocity $\mathrm{V}_{\text {Feed }}$ and the $\Delta \mathrm{T}_{\text {furnace }}$ that is defined as the difference between $\mathrm{T}_{\text {feed }}$ and $\mathrm{T}_{\text {growth }}$. The combination of these two parameters $V_{\text {Feed }}$ and $\Delta T_{\text {furnace }}$ leads to different configurations:

i) when the melt is fed quickly into the growth crucible $(10 \mathrm{~mL} / \mathrm{min}<$ $\mathrm{V}_{\text {feed }}<20 \mathrm{~mL} / \mathrm{min}$ ), the configuration is named as "Casting".

ii) if the drops of feed material impact directly on solid, the configuration is named as "Splat Cooling".

iii) when the combination of $\mathrm{V}_{\text {feed }}$ and $\Delta \mathrm{T}_{\text {furnace }}$ results in a small melt in contact with the growing solid, the configuration is named as "Feeding".

Each acronym has been chosen for its similarity with the original technique name and due to the number of experimental configurations used to synthesis the materials, a nomenclature has been defined as reported in Table 1. For simplicity, each acronym of Table 1 is followed by the material characteristics, i.e (Un) for undoped $\mathrm{GaSb}$, (Te) for Te-doped $\mathrm{GaSb}$ or (In-) for GaInSb alloys, in this case the digits correspond to the nominal concentration $\left(\mathrm{C}_{0}\right)$ in at $\%$. As an example, an experiment performed with a $\mathrm{V}_{\text {feed }}$ of $10 \mathrm{~mL} / \mathrm{min}$ and a $\Delta \mathrm{T}_{\text {furnace }}$ of $120 \mathrm{~K}$ with undoped $\mathrm{GaSb}$ is named LC-Un while an experiment carried out with a $12 \%$ at GaInSb alloy using a $V_{\text {feed }}$ of $1 \mathrm{~mL} / \mathrm{min}$ and a $\Delta \mathrm{T}_{\text {furnace }}$ of $320 \mathrm{~K}$ is labeled LSp-In12. 


\section{PRINCIPAL RESULTS}

In previous works, we applied our experience in bulk growth by the Vertical Bridgman technique to study the quality of polycrystalline Te-doped GaSb materials, showing that the solute concentration along the growth axis is typically described by a Scheil law and that an increase of the pulling velocity and solute concentration leads to higher degree of polycrystallinity [2]. Our experience in bulk growth of GaInSb materials prepared by the Vertical Bridgman technique showed that the In concentration increase along the growth axis following a Scheil law [11] leading to a low yield for the production of substrates with high In content.
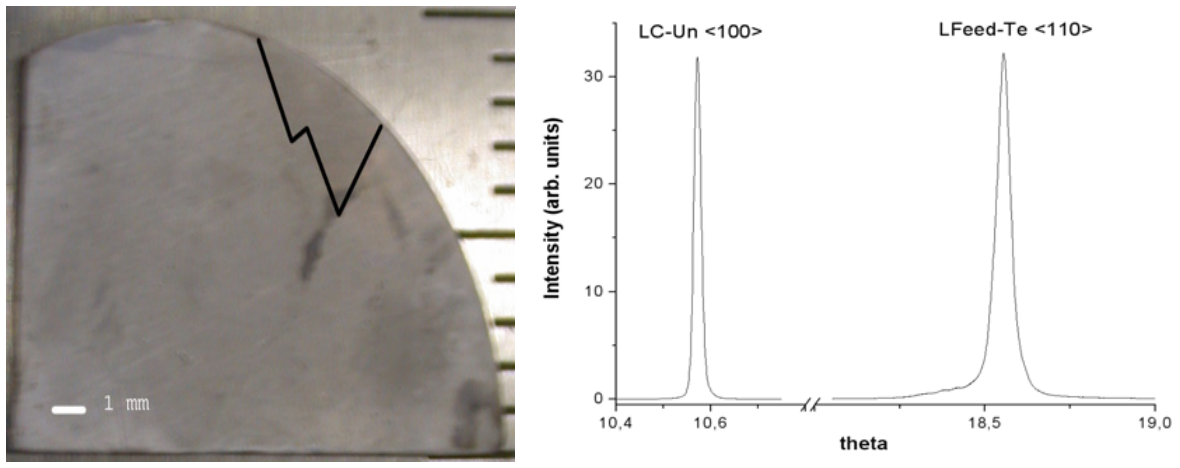

FIGURE 3. Single LFeed-Te crystal (a) and LC-Un and LFeed rocking curves (b).

In the case of the VFM, experiments performed with the Low Casting configuration for undoped and Te-doped GaSb [12] showed that for undoped materials (LC-Un), the samples were almost monocrystallines while Te-doped samples (LC-Te) showed a high degree of polycrystallinity with nearly all the orientation expected for $\mathrm{GaSb}$ appearing on the XRD spectra. In addition, the segregation profile along vertical axis of Te-doped materials showed an inverted profile in comparison with the Scheil law. These results demonstrated that monocrystals can be produced by VFM and suggested that segregation control may be also achievable. Using the High Casting, High Feeding and Low Feeding configurations [13], Te-doped materials with a homogenous solute content have been effectively obtained. The samples showed different grain structures and sizes with average values ranging from $0.5 \mathrm{~mm}$ for HC-Te samples to several $\mathrm{mm}$ for LFeed-Te samples, as shown on figure 3a, demonstrating that monocrystals can also be achieved for Te-doped GaSb prepared by the VFM. The structural, electrical and luminescence properties of the single crystals obtained with the VFM are summarized in the following paragraph.

Rocking curve and Laue diagram have been used to orientate and characterize LCUn and LFeed-Te single crystals. Laue diagrams demonstrated that LC-Un samples have an orientation close to $\langle 111\rangle$ while LFeed-Te samples generally exhibit a misorientation of several degrees from the $<110>$ direction. The quality of both materials has been confirmed by the high symmetry of the rocking curves reported on figure $3 b$. The Full Width at Half Maximum (FWHM) of LC-Un samples is about the 
same as the one obtained for commercial wafers [14] while the FWHM of LFeed-Te samples is about four times larger than for LC-Un samples.

The electrical characteristics obtained by Hall measurements at room temperature [12] showed that LC-Un samples exhibits a p-type conduction, with a carrier density (p) of $3 \cdot 10^{17} \mathrm{~cm}^{-3}$, a resistivity $(\rho)$ of about $0.07 \Omega \cdot \mathrm{cm}$ and a mobility $(\mu)$ of about 300 $\mathrm{cm}^{2} \cdot \mathrm{V}^{-1} \cdot \mathrm{s}^{-1}$. In the case of LFeed-Te samples, measurements showed a n-type conduction with a carrier density of $1 \cdot 10^{18} \mathrm{~cm}^{-3}$, a resistivity of about $0.0038 \Omega$.cm and a mobility of $1500 \mathrm{~cm}^{2} \cdot \mathrm{V}^{-1} \cdot \mathrm{s}^{-1}$.
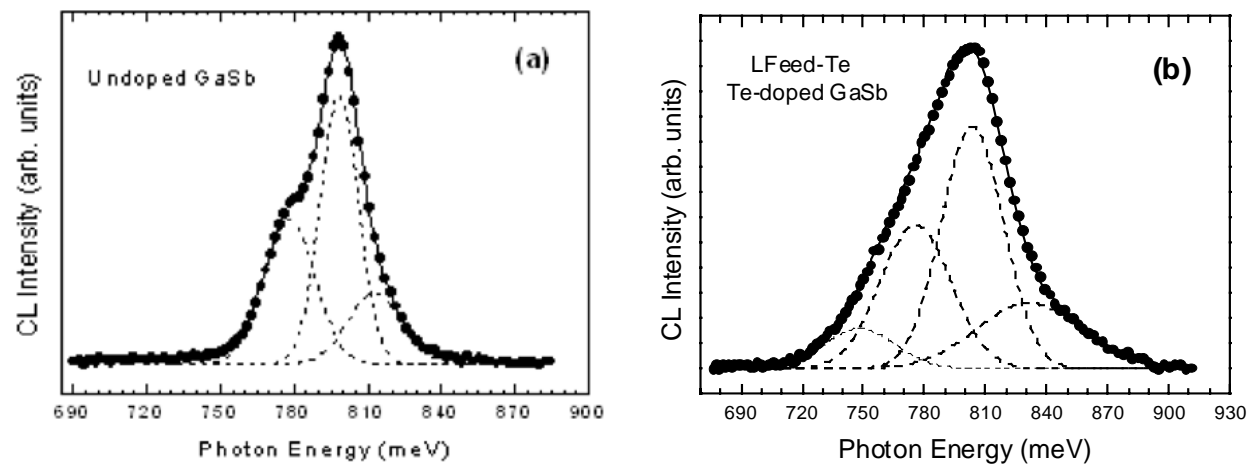

FIGURE 4. Gaussian deconvolution of CL spectra obtained for LC-Un (a) and LFeed-Te (b) single crystals.

The luminescence properties were studied through Cathodoluminescence at $80 \mathrm{~K}$ following the procedure of Ref. [12]. The spectral distribution of the CL emission of both LC-Un and LFeed-Te samples showed a quite homogenous spatial luminescence. CL spectra of LC-Un samples appeared always peaked at about $795 \mathrm{meV}$ and the Gaussian deconvolution of such spectra (figure $4 \mathrm{a}$ ) reveals the existence of three emission bands centered at about 812, 797 and $777 \mathrm{meV}$ corresponding to tail states, band edge and band A respectively. For LFeed-Te samples, CL measurements also revealed a quite homogenous luminescence spatial distribution with the existence of three emission bands peaked at 843, 811 and $775 \mathrm{meV}$ (figure 4b). The emission peaked at $811 \mathrm{meV}$ corresponds to the GaSb-Te near band-edge luminescence, shifted towards higher energies by the Moss-Burstein effect $[15,16]$. The band peaked at 773 meV corresponds to the A band while the transition centered at about $841 \mathrm{meV}$ was tentatively attributed to a transition involving a Te-related level located below the second minimum of the conduction band and is probably due to the Te concentration [17]. Under low excitation conditions, a weak emission band peaked at about $745 \mathrm{meV}$ corresponding to band $\mathrm{C}$ was also revealed.

All these measurements demonstrated a quite good homogeneity of the single crystals properties produced by the VFM for both undoped and Te-doped GaSb materials.

In the case of concentrated GaInSb alloys, experiments performed with the Low Casting configuration for alloys with a $\mathrm{C}_{0}$ of $10 \%$ at (LC-In10) [18], showed a low 
polycrystalline behavior with an almost homogenous In distribution. Similarly to LCTe experiments, an inverted solute segregation profile was found along the growth axis with an average concentration of about 10 at $\%$ for samples extracted from the bottom of the ingot and of about 2 or $3 \%$ at for samples extracted from the middle and from the top. Thermal measurements performed during LC-In10 experiments showed that the low In content material obtained for middle and top samples can be attributed to a directional solidification without important effect of the Constitutional Supercooling [18].
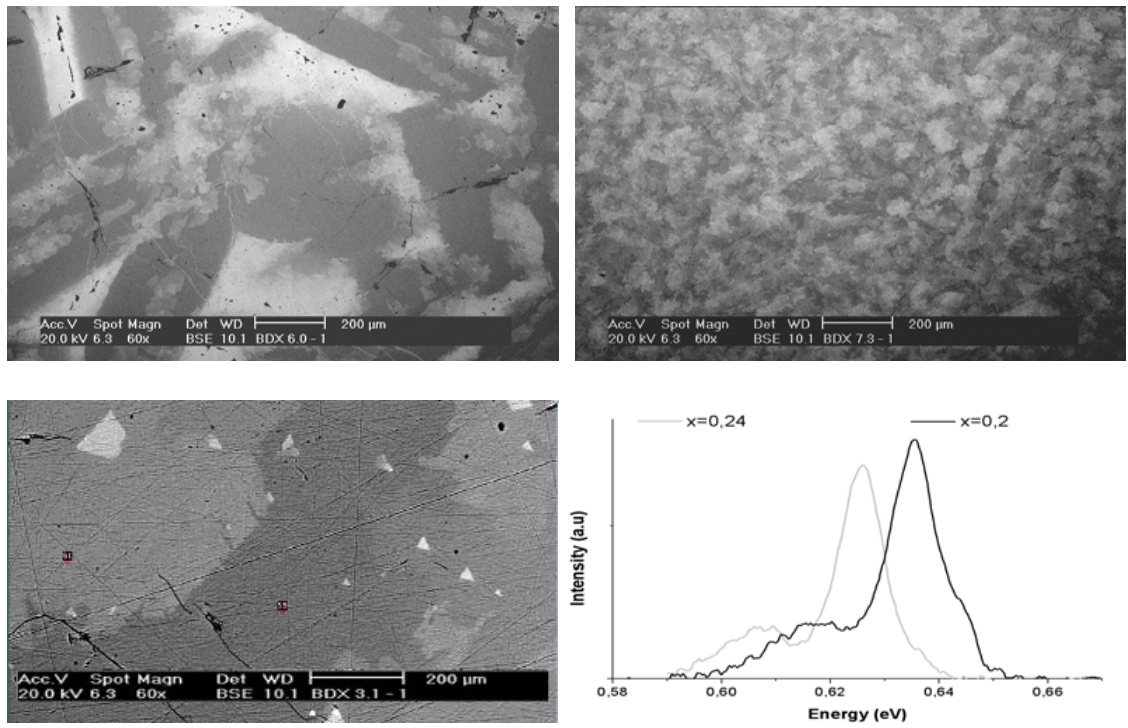

FIGURE 5. BSE micrographs showing different grey levels corresponding to the different In spatial distribution of HC-In12 (a), LSp-In12 (b) and LFeed-In15 (c) samples, in all cases the average In content ranges between 10 and $12 \%$ at. PL spectra of LFeed-In15 (d) samples.

In contrary, using the High Casting configuration, solid solutions with an average In content close to the nominal one were obtained. As shown on the Back Scattered Electrons (BSE) micrograph of a HC-In12 experiment presented on figure 5a, the samples were typically composed by a matrix of about $1-2$ at $\%$ In, corresponding to the extended dark grey areas, and precipitates of 30 at $\%$ to 50 at $\%$ of In which correspond to the white grey areas. This microstructure is typical of quenching experiments, and thermal measurements showed that it can be attributed to the nucleation and unidirectional growth of crystallites into a low undercooled melt [18].

The experiments performed in the casting configuration indicated that in both configurations, the production of a microstructure leads to solid solutions with a high average In content.

In order to study the microstructure production, splat cooling experiments were performed with concentrated alloys of different concentrations 12,10 and 2.5 at $\%$ [19]. Similarly to HC-In12 experiment, all the splat cooling samples showed an average In concentration close to the nominal one. However, as shown on figure 5b, 
where a typical BSE micrograph of LSp-In12 experiments is reported, the samples exhibited a refined microstructure in comparison with HC-In12 ones. To characterize the microstructures obtained in such experiments, a characteristic length $\left(l_{M}\right)$ was defined as the average length between the centre of an area with low In content $\left(\mathrm{A}_{0}\right)$ and the centre of a neighboring area with high In content $\left(\mathrm{A}_{\infty}\right)$. The results indicated that $l_{M}$ increases as $C_{0}$ is reduced which is in agreement with the general interface stability theory of Mullins-Sekerka (MS) [20]. This theory that relates the interface stability with the interface velocity and concentration predicts that for a given concentration, the instability wavelength that can develop on the Solid/Liquid interface should increase as the interface velocity is reduced, leading to more extended microstructures.

In order to reach lower interface velocity, Low Feeding experiments were carried out, using a low $\mathrm{V}_{\text {feed }}$ of $0.1 \mathrm{~mL} / \mathrm{min}$ with a $\Delta \mathrm{T}_{\text {furnace }}$ of $120 \mathrm{~K}$ [19]. The compositional study of samples obtained from experiments performed with a nominal concentration of 15 at $\%$ (LFeed-In15) showed (figure 5c) an extended microstructure with a grey matrix of about 7-10 at $\%$ In, with smaller areas of 3-4 at $\%$ In and inclusions with average size of $50 \mu \mathrm{m}$ with a typical In concentration of $20-22$ at $\%$. The average concentration was comprised between 10 and 12 at $\%$ and both XRD and etching of the LFeed-In15 samples indicated a low polycrystalline behavior. The band edges estimated from PL measurements at $4 \mathrm{~K}$, was about $635-640 \mathrm{meV}$ which correspond to a concentration of 10-12 at \% in agreement with the results reported by Allegre et al. for GaInSb samples [21]. On figure $5 \mathrm{~d}$ is reported the spectra recorded for samples of 10 and 12 at\% which demonstrate the effective band edge variation with the In concentration.

The results obtained with the Low Feeding indicate that materials with high In content and effective band edge reduction can be prepared with this configuration and suggest that increased homogeneity may be achieved by optimizing the feeding rate and $\Delta \mathrm{T}_{\text {furnace }}$ combination.

\section{CONCLUSIONS}

In this work, the different elements of the Vertical Feeding Method (VFM) set-up have been detailed and the principal results obtained for GaSb and GaInSb materials have been summarized. The study demonstrated that segregation can be controlled by this technique and that single crystals can be obtained for GaSb and Te-doped GaSb alloys. In the case of GaInSb alloys, In-rich solid solutions with different In spatial distribution have been obtained with the different VFM configurations and the Low Feeding configuration seems to be the most appropriate to reach effective band edge reduction and higher spatial homogeneity.

\section{ACKNOWLEDGMENTS}

This work has been carried out in the frame of the Fifth Framework European Programme for research, HPRNCT 2001-00199 project. Support from MCYT through 
projects, MAT 2003-09873, ESP 2004-0041-E and MAT2003-00455 is also acknowledged.

\section{REFERENCES}

1. O.V. Sulima, A.W. Bett, P.S. Dutta, H. Ehsani and R.J. Gutmann, Proceedings of the 16th European PV Solar Energy Conference,VA2.24, Glasgow (UK), 2000.

2. J. Vincent, D. Martín, V. Bermúdez, C. Algora and E. Diéguez, Proceding of TPV6, AIP Conf. Proc. 738,368 (2004).

3. A. Mitric, T.Duffar, C. Diaz-Guerra, V. Corregidor, L. C. Alves, C. Garnier, G. Vian J. Cryst. Growth 287, 224-229 (2006).

4. Dutta, P.S., and Ostrogorsky, A.G., J. Cryst. Growth 194, 1-7 (1998).

5. Tanaka A., A. Watanabe, M. Kimura, T. Sukegawa, J. of Crystal Growth 135, 269-272 (1994).

6. A. Mitric, J. Vincent, R. Caillard, C. Stelian, V. Bermudez, E. Dieguez and T. Duffar Proceding of TPV6. AIP Conf. Proc. 738, 377 (2004).

7. O. Bonino, P. De Rango, R. Tournier, J. of Magnetism and Magnetic Materials 212 (2000) 225-230.

8. S Goda, T. Moratani, Y. Hatanka, H. Shimizu and I. Hide IEEE First World PV Conf. (1994).

9. G. Dour, E. Ehret, A. Laugier, D. Sarti, M. Garnier, F. Durand J.Crystal Growth 193 (1998) 230.

10. J. Vincent, V Bermudez, E. Dieguez. Eur. J. Appl. Phys. 27, 419-421 (2004)

11. J. Vincent, V. Bermudez, E. Dieguez, L.C. Alves, V. Corregidor, N.P. Barradas J. Cryst. Growth 275 (2005) e537-e542.

12. J. Vincent, C. Díaz - Guerra , J. Piqueras, A. Amariei, E.K. Polychroniadis and E. Dieguez. J. Cryst. Growth 289 (2006) 18-23.

13. J. Vincent, C. Díaz - Guerra , J. Piqueras and E. Dieguez. J. Cryst. Growth 293, (2006) 285-290.

14. GaSb samples supplied by Wafer Technology Ltd.

15. T.S. Moss, Proc. Phys. Soc. London Sect. B 76 (1954) 775.

16. E. Burstein, Phys. Rev. 93 (1954) 632.

17. A.S. Filipchenko, L.P. Bolshakov, A. Naurizbaev, A.G. Braginskaya, A.N. Popov, phys. stat. sol. (a) 48 (1978) K115.

18. J. Vincent, E. Dieguez Accepted for publication J. Cryst. Growth.

19. J. Vincent, E. Dieguez Sent for publication J. Opto. Adv. Mat.

20. R. Trivedi, W.Kurz, Acta Metal. 34 (1986) 1663.

21. J. Allegre, M. Averous, R. Jourdain, A. Joullie Journal of Luminescence 11 (1976) 339-347. 
Copyright of AIP Conference Proceedings is the property of American Institute of Physics and its content may not be copied or emailed to multiple sites or posted to a listserv without the copyright holder's express written permission. However, users may print, download, or email articles for individual use. 